\title{
Revisiting the Relationship Between Electrogustometry and Sour Taste Perception
}

\author{
Jennifer A. Stillman* \\ Massey University Albany, Private Bag 102-904 North Shore MSC, Auckland, New Zealand
}

\begin{abstract}
In a recent study Ellegård, Goldsmith, Hay, and Morton concluded that electrogustometry is neither mediated by sour taste receptors, nor reflects sour taste quality. While aspects of electric taste await a full explanation, such farreaching claims have significant implications for the future of electrogustometry in clinical practice. The current paper presents a critique of the grounds upon which the arguments were based, and suggests that these conclusions may be premature.
\end{abstract}

Ellegård, Goldsmith, Hay and Morton [1] addressed the contention that taste perception with anodal electrogustometric stimulation is mediated via sour taste receptors. Their stated aim was to evaluate "to what extent electrogustometry relates to an ability to detect sour taste - rather than sweet, salt, or bitter." They reasoned that if anodal stimulation activates sour taste receptors, then the positive predictive values of electrogustometry should be better for sour taste perception than for other taste qualities. They correlated electrogustometric thresholds with visual analogue scale (VAS) ratings to whole mouth and regional applications of taste solutions provided by patients with various disorders that have the potential to affect taste. Because the pattern of predictive values did not favour sour taste they rejected the view that "electrogustometry is mediated by sour taste receptors or even that it reflects the sour taste quality" [1]. Instead they concluded that electrogustometry measures a function of taste perception different from that induced by chemical stimuli.

Research has yet to provide definitive answers to some of the questions that are raised concerning electric taste. In the meantime the validity of the arguments upon which conclusions are based must be established, because conclusions such as those reached by Ellegård et al. have significant implications for the future of electrogustometry in clinical practice. Here I challenge the expectation that electrogustometric thresholds should be better predictors of VAS scores from sour-tasting stimuli than from other solutions.

\section{ELECTRICAL TASTE, SOUR TASTE, AND pH}

It may be tempting to overlook the fact that although an electrogustometer provides a quantitative threshold estimate; the device does not directly measure a taste response. Instead it delivers a stimulus. The ensuing perceptual response is quantified using an operational definition: namely, the current required for a chosen percentage of stimulus detections. Classically the stimulus delivered via electrogustometry was

*Address correspondence to this author at the Massey University Albany, Private Bag 102-904 North Shore MSC, Auckland, New Zealand; Tel: 649 4140800; Fax: 649414 0831; E-mail: J.A.Stillman@massey.ac.nz referred to as an "adequate" stimulus in view of the liberation of protons in the vicinity of the anode [2]. Acids are, of course, proton donors, and their association with sour taste is reflected in the Latin root of the word acid meaning "to be sour". Sour or soapy tastes have been reported, depending upon polarity, both with the use of electrogustometric stimuli, and if a battery is licked. Grant, Ferguson, Strang, Turner and Bone [3] demonstrated a linear relationship between current and $\mathrm{pH}$ at the active electrode such that the anode lowers $\mathrm{pH}$ (increases the concentration of hydrogen ions), and the cathode raises $\mathrm{pH}$ (increases the concentration of hydroxyl ions), creating respectively a locally acidic or alkalinic environment. Therefore with anodal current sour taste thresholds are specified as the current strength required for a given percentage of stimulus detections. No such theoretical underpinning allows electrogustometry to be associated with tastes evoked by sweet or bitter stimuli. If the current is increased beyond a certain level [4] sensations such as stinging and tingling are produced suggesting direct stimulation of the taste receptors and/or activation of the trigeminal system. The latter might occur by two routes, direct neural activation, or activation as a result of the acid itself.

The mechanisms underlying responses to both galvanic and acidic stimulation have been explored in diverse ways. The relationship between the number of fungiform papillae and responses to electrogustometric stimulation was examined by Miller, Mirza and Doty [5] who obtained support for the proposal that sensations arising from current levels employed in electrogustometric testing arise from the taste system. Such a conclusion is also supported by Niyomia and Funakoshi [6] who found that $\mathrm{FeCl}_{3}$ which depolarizes the cells in taste buds, obliterates electrical taste responses in rats. Kinnamon [7] reviewed studies revealing that ionic taste stimuli, such as salts and acids, interact directly with ion channels to depolarize taste cells. In contrast, amino acids, sweeteners, and most bitter tasting stimuli, were found to bind to specific membrane systems. DeSimone, Lyall, Heck and Feldman [8] acknowledged acid stimuli as the unique sources of sour taste, in an investigation of the mechanisms by which acidic stimuli activate taste receptors by means of pH-tracking. 
Electrogustometric data and those from conventional taste stimuli are usually distinguished by the manner in which the stimuli were delivered. Stevens, Baker, Cutroni et al. [9] eliminated this difference in a study in which all taste stimuli, whether electrical or conventional, were delivered via a metal delivery stem. Their results were consistent with the electrolytic chemical hypothesis, and when electrical and taste stimuli were compared, metallic, sour-bitter, and sour tastants and descriptors, were perceptually closest to electrical stimuli, and described anodal stimulation better than cathodal.

It is unfortunate that a reference to a classical and still relevant chapter by Bujas [10] in Ellegård et al. [1] manuscript implied particular support for the direct-effect hypothesis. In fact, that chapter evaluated both the electrolytechemical and the direct-effect hypotheses in some detail, and tended to favour the former. Bujas cites research suggesting that the anodal sour taste is mainly the effect of electrolysis, but an undefined cathodal taste is the effect of a direct action of the current, and is associated with a bitter cathodal taste that is the result of electrolysis. In a later paper by Bujas [2] those same conclusions are reiterated. Bujas [10] does however acknowledge that, in special circumstances "electric current might act for the most part directly as an inadequate stimulus" (italics theirs). But even under the specified circumstances he suggests that the current probably acts on receptor cells rather than on nerve fibres.

\section{RECEPTOR ACTIVATION AND TASTE PERCEP- TION}

Theories of taste perception have a bearing on whether stronger correlations would be expected between electrogustometric thresholds and intensity ratings to regional or whole mouth acid solutions than to other solutions. This would be true whatever receptors were activated by the electrical stimulus.

In moving from a contention about the activation of sour taste receptors to a discussion of sweet, sour, salty, or bitter taste perceptions, Ellegård et al. [1] implicitly assume a labelled-line, rather than an across-fibre, theory of taste perception. Both have been the subject of in-depth discussions in a recent article by Erikson [11], published along with associated peer commentaries.

The across fibre pattern theory of taste arguably has stronger support than a strictly labelled line approach [11]. For example, evidence exists that there are several signal pathways that respond in each taste category (see Lindemann [12] for a review). Therefore, with the possible exception of rare cases involving faulty genes for particular receptors, or for second messengers; or medications having particular molecular-level effects, it is unlikely that an enduring diminution of taste experience is associated with the loss of one particular taste quality. The majority of clinical cases exhibiting taste loss will be attributable to disorders affecting the peripheral or central neural pathways for taste, thus affecting taste as a whole. In the case of Ellegård et al. [1] diverse patient population, medication is an extraneous variable, which may have had some potential to influence intensity ratings to particular solutions. However, apart from the exceptions mentioned above, if taste is affected across the board, responses to any one taste quality provide an indica- tion of the condition of the taste system overall. Therefore, although electrogustometry can be used to activate only sour taste responses, these can stand proxy for responses to other taste qualities, none of which would be expected a priori to correlate more highly with electrogustometric thresholds than another. Similarly, where there is general taste nerve damage, regional testing with, for example, sucrose, might reveal a diminution that would correlate as well with wholemouth responses to salt, sour, or bitter solutions as with those to sweet.

\section{INTER-TASTANT COMPARISONS}

Ellegård et al. [1] acknowledge that an overall cut-off at $>=$ VAS 50 for normal taste may be questionable. In this regard a number of studies reviewed by Frank, Hettinger \& Clive [13] established significant differences in intensity ratings among different age groups. This may be due, in part, to the fact that taste bud density is known to vary by age [14, 15], and many of the subjects in Ellegård et al. 's [1] study were older-aged, so that the chosen cut-off may have placed normal aged individuals into a non-normal category. In any case, a lack of correlation between threshold measures and supra-threshold intensity ratings [16] is well known, and is in keeping with the non-significant whole mouth correlations reported by Ellegård et al. [1]. Perhaps the small and fairly comparable correlations in the expected direction with regional testing reflect the smaller number of taste fibres recruited, making the reported taste experience less distant from the electrogustometric threshold to which they were compared. Unfortunately a large proportion of the VAS scores used in the analyses were at ceiling, while many of the electrogustometric thresholds were at the floor set by the limit of the electrogustometer. These range restrictions would serve to further reduce any correlations.

It is reasonable to suppose that threshold sensitivity will depend upon the number of taste receptors activated. Miller and Reedy [15] established that, indeed, differences in taste sensitivity among normal subjects are associated with differences in taste bud densities. They also examined taste intensity ratings for four suprathreshold concentrations of sucrose, $\mathrm{NaCl}$, PROP, citric acid, and quinine $\mathrm{HCl}$ applied to the tongue tip with a cotton-tipped applicator. The subjects were divided into two groups $(\mathrm{N}=8$ each) based on their taste bud densities. The series of concentrations of sucrose, $\mathrm{NaCl}$, and citric acid included identical concentrations to those used by Ellegård et al. [1]. The concentration of their bitter exemplar, quinine, was the same as Ellegard's bitter exemplar, caffeine, although whether the identical concentrations of these contrasting bitter substances produced comparable intensity ratings is unknown. Miller and Reedy's [15] higher and lower taste pore density groups did not differ significantly in their intensity ratings for citric acid or quinine, but differed significantly for $\mathrm{NaCl}$ and sucrose. Figure 3 in that publication suggests that, at the highest concentration, as employed in Ellegård et al. [1] study, $\mathrm{NaCl}$, sucrose and quinine produced the highest intensity ratings from those with lower taste bud densities, while citric acid produced equivalent ratings for the two groups. Overall, were the two groups to be combined, the highest intensity ratings would be for $\mathrm{NaCl}$, as in Ellegard's study. The study also established that the slopes of the functions relating perceived intensity to concentration differed among compounds. If the slope of the intensity 
function varies for different taste bud densities, different patient groups, or different tastants, then the correlation between a single electrogustometric threshold and suprathreshold VAS scores for various solutions would be expected to be weak, and may or may not vary together, irrespective of whether taste responses are depressed across the board.

\section{CONCLUSION}

By itself the connection between hydrogen ions and both sour and electric taste does not seem controversial. The advantage of electrogustometry is that it can deliver a repeatable stimulus to obtain an objective threshold estimate. Everyday foods and beverages, however, produce a perceptual experience based on intricate activations over myriad receptors. Both electric stimuli and single tastant solutions represent an attempt to restrict taste activation to one class of receptor. Electrogustometry is specifically a threshold procedure, and in common with thresholds obtained using solutions, does not correlate well with supra-threshold responses in individuals with normal taste. To expect a higher positive predictive value of electrogustometry for supra-threshold sour taste perception is to make an a priori assumption that, in individual patients, suprathreshold responses to various categories of solution do not covary. If they do covary, any predictive value of electrogustometry for sour taste will represent other tastes as well, validating its use in clinical and research applications.

\section{REFERENCES}

[1] Ellegård EK, Goldsmith D, Hay D, Morton RP. Studies on the relationship between electrogustometry and sour taste perception. Auris Nasus Larynx 2007; 34: 477-80.
[2] Bujas Z. Sensory effects of continuous and repetitive electrical stimulation of the tongue. In: Le Magnen J, MacLeod P, Eds. Proceedings of the Sixth International Symposium on Olfaction and Taste, Gif-sur-Yvette, Paris. London \& Washington DC: Information Retrieval: 1977; pp. 265-71.

[3] Grant R, Ferguson MM, Strang R, Turner JW, Bone I. Evoked taste thresholds in a normal population and the application of electrogustometry to trigeminal nerve disease. J Neurol Neurosurg Psychiatry 1987; 50: 12-21.

[4] Stillman, JA, Morton RP, Hay KD, Ahmad Z, Goldsmith D. Electrogustometry: Strengths, weaknesses, and clinical evidence of stimulus boundaries. Clin Otolaryngol 2003; 28: 406-10.

[5] Miller SL, Mirza N, Doty RL. Electrogustometric thresholds. Relationship to anterior tongue locus, area of stimulation, and number of fungiform papillae. Physiol Behav 2002; 75: 753-57.

[6] Ninomiya Y, Funakoshi M. Role of ions in generation of taste nerve responses to electrical tongue stimulation in rats. Jpn J Physiol 1981; 31: 891-902.

[7] Kinnamon SC. Taste transduction: Linkage between molecular mechanisms and psychophysics. Food Qual Prefer 1996; 7: 153-59.

[8] DeSimone JA, Lyall V, Heck GL, Feldman GM. Acid detection by taste receptor cells. Respir Physiol 2001; 129: 231-45.

[9] Stevens DA, Baker D, Cutroni E, Frey A, Pugh D, Lawless HT (2008). A direct comparison of taste of electrical and chemical stimuli. Chem Senses 2008; 33: 405-13.

[10] Bujas Z. Electrical taste. In: Autrum H, Jung R, Loewenstein W, MacKay D, Teuber H, (Eds). Handbook of sensory physiology, Berlin/Heidelberg/New York: Springer-Verlag: 1971; pp. 180-99.

[11] Erickson RP. A study of the science of taste: On the origins and influence of core ideas. Behav Brain Sci 2008; 31: 59-91.

[12] Lindemann L. Taste reception. Physiol Rev 1996; 76: 719-66.

[13] Frank ME, Hettinger TP, Clive JM. Current Trends in Measuring Taste. In: Doty RL, Ed. Handbook of Olfaction and Gustation, Basel, Hong Kong NYU, Marcel Dekker Inc 1995; pp. 669-88.

[14] Miller IJ Jr. Human taste bud density across adult age groups. J. Gerontol Biol Sci 1988; 43: B26-30.

[15] Miller IJ Jr, Reedy FE Jr. Variations in human taste bud density and taste intensity perception. Physiol Behav 1990; 47: 1213-9.

[16] Bartoshuk L. Clinical evaluation of the sense of taste. Ear Nose Throat J 1989; 68: 331-7. 